\title{
ADAPTIVE FUZZY SLIDING MODE CONTROLLER FOR THE SNORKEL UNDERWATER VEHICLE
}

\author{
Eduardo Sebastián \\ Lab. de Robótica y Exploración Planetaria, Centro de Astrobiología, Ctra. Ajalvir Km.4, Torrejón de Ardoz, Spain \\ sebastianme@inta.es \\ Miguel A. Sotelo \\ Universidad de Alcalá. Departamento de Electrónica. Ctra. N-II Km. 33. Alcalá de Henares. Madrid, Spain \\ miguel.sotelo@uah.es
}

\begin{abstract}
Keywords: $\quad$ Sliding mode control, fuzzy models, nonlinear control, robots dynamics, marine systems.
Abstract: This paper describes a control system for the kinematic variables of an underwater vehicle. Control of underwater vehicles is not simple, mainlys due to the nonlinear, coupled and unknown character of system equations and dynamics. The proposed methodology makes use of a pioneering algorithm implemented for the first time in an underwater vehicle, and it is based on the fusion of a sliding mode controller and an adaptive fuzzy system, including advantages of both systems and relaxing the required knowledge of vehicle model.
\end{abstract}

\section{INTRODUCTION}

The adaptive control of nonlinear systems, with model and parameter uncertainty, has been a focus of research, in which important progress has been obtained in the last years (Gee, et al., 1999; Wang, et al., 2000). Several kinds of control systems for underwater vehicles have been proposed in the literature. From sliding controllers (Yoerger and Slotine, 1991), adaptive nonlinear MIMO controllers in (Antonelli, et al., 2003; Choi and Yuh, 1996), to those based on intelligent control (Yuh, 1994; DeBitetto, 1994). In (Fossen, 1994), the reader can find a review of some of these methods. In spite of that, the design of a unique controller for all the DOF of an underwater vehicle is an area of research still open (Yuh, 2000).

The controller proposed in this paper is based on the work and results developed in (Wang, et al., 2000), about adaptive fuzzy sliding mode control, spreading the results to a MIMO controller. The controller performance is analyzed in a practical application where 4 DOF must be controlled, in the underactuated UUV, called Snorkel.

The controller is part of a control architecture, which is based on three chained controllers (propulsion, kinematic variables and vehicle guidance), whose goal is to generalize the system dynamics for their use by the controllers at a higher hierarchical level (Espinosa, et al., 1999). So the guidance system, using local environment information, provides the input references to the kinematic variables controller, in order to navigate in a horizontal plane (surge and yaw velocities).

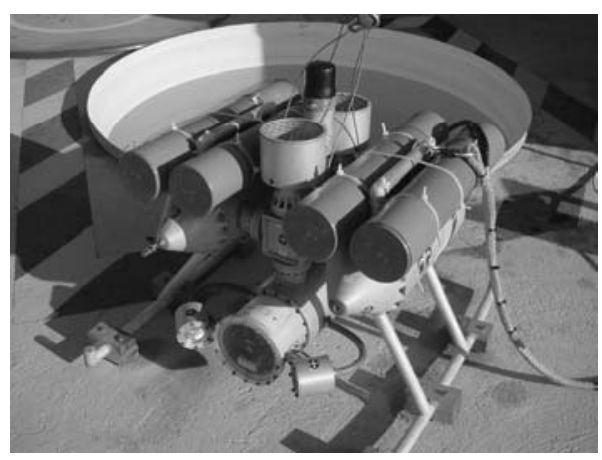

Figure 1: Snorkel robot image

The paper is organized as follows; section 2 introduces the dynamic equations of the Snorkel underwater vehicle. In section 3 the adaptive fuzzy sliding mode controller is presented. In section 4 real test results are presented, and the controller 
performance is described. Finally, section 5 summarizes the results.

\section{VEHICLE DYNAMICS}

In this work, a Newton-Euler formulation and a noninertial reference frame have been selected, as the method to represent vehicle dynamics (Fossen, 1994). Specifying dynamic equations for the Snorkel vehicle (1) and its four controllable DOF (surge, heave, pitch and yaw), and using a change of variable for the driver vector, allows the designer to decouple the input control of each thrusters

$$
M v_{S}+C(v) v_{S}+D(v) v_{S}+g(\eta)-g\left(\tau_{S}\right)=H \tau_{S},
$$

where $\boldsymbol{M}$ is the diagonal matrix of inertia $(4 \times 4)$; $\boldsymbol{D}(\boldsymbol{v})$ is a diagonal matrix $(4 \times 4)$ that contains damping coefficients; $\boldsymbol{g}\left(\boldsymbol{\tau}_{S}\right)$ represents the thrust perturbation vector of coupled terms $(4 \times 1) ; \boldsymbol{g}(\boldsymbol{\eta})$ is a vector $(4 \times 1)$ that includes buoyancy and weight forces; $\boldsymbol{C}(\boldsymbol{v})$ is the Coriolis and centripetal matrix $(4 \mathrm{x} 4) ; \boldsymbol{H}$ is a diagonal driver matrix $(4 \mathrm{x} 4) ; \boldsymbol{v}$ is the velocity vector and $\boldsymbol{v}_{S}$ is the velocity vector in its controllable DOF, both relative to fluid and in a body-fixed frame and $\boldsymbol{\tau}_{S}$ is the thrust vector.

\subsection{Affine System in Controllability Canonical Form}

In order to develop the controller proposed, the equations of the vehicle must be expressed in controllability canonical form. Assuming high stability in pitch and roll movements, this representation is possible.

If the change of nomenclature $\boldsymbol{x}=\boldsymbol{v}_{S}$ and $\boldsymbol{u}=\boldsymbol{\tau}_{S}$ is done, where $\boldsymbol{x}$ is the new state vector and it represents the angular and linear position of the vehicle in a body-fixed reference frame, the value of

$\boldsymbol{x}$ extracted, and system (1) can be represented in the controllability canonical form.

$$
\boldsymbol{x}^{(1)}=\boldsymbol{x}^{(2)}, \quad \boldsymbol{x}^{(2)}=\boldsymbol{f}(\xi)+\boldsymbol{g}(\xi) \boldsymbol{u},
$$

\section{ADAPTIVE FUZZY SLIDING MODE CONTROL}

This section studies the properties of the sliding controller and fuzzy estimators.

\subsection{Sliding Mode Controller}

Considering the control problem of a system like (2), and the definition of the sliding surface (3)

$$
s=\dot{\tilde{x}}+\Lambda \tilde{\boldsymbol{x}}
$$

where $\tilde{\boldsymbol{x}}=\boldsymbol{x}-\boldsymbol{x}_{d}, \boldsymbol{x}_{d}$ is the input reference vector and $\boldsymbol{\Lambda}$ is a positive definite matrix. The sliding control theory (Slotine and $\mathrm{Li}, 1991$ ) proposed the control law (4), and ensures that after reaching the surface $\boldsymbol{s}=0$ the system follows the input references in presence of uncertainties, if $\boldsymbol{s}^{T} \boldsymbol{s} \leq-\boldsymbol{\eta}^{T}|\boldsymbol{s}| \quad \boldsymbol{\eta} \geq 0$.

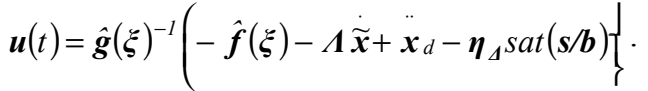

where $\boldsymbol{\eta}_{\Delta}[l]_{4 \times 1} \geq \boldsymbol{\eta}^{T}, \hat{\boldsymbol{g}}(\boldsymbol{\xi})$ and $\hat{\boldsymbol{f}}(\boldsymbol{\xi})$ are a set of fuzzy estimators of the partially unknown and nonlinear functions $\boldsymbol{f}(\boldsymbol{\xi})$ and $\boldsymbol{g}(\boldsymbol{\xi})$, and $\operatorname{sat}(\boldsymbol{s} / \boldsymbol{b})$ is a saturation function that uses a boundary layer $\boldsymbol{b}$, close to the sliding surface, to eliminate the chattering effect (Slotine and Li, 1991).

\subsection{Fuzzy Adaptive Systems}

A fuzzy system may be used like a nonlinear universal approximator (Wang, et al., 2000), due to its ability to introduce verbal information, and its capacity to uniformly approximate any real and continuous function with different degrees of precision. So functions $f_{i}(\xi)$ and $g_{i}(\xi)$, of the $i^{\text {th }}$ controllable DOF, are parameterized by fuzzy systems as

$$
\begin{aligned}
& \hat{f}_{i}\left(\boldsymbol{\xi} \mid \boldsymbol{\theta}_{f i}\right)=\boldsymbol{\theta}_{f i}{ }^{T} \boldsymbol{\zeta}(\boldsymbol{\xi}), \\
& \hat{g}_{i}\left(\boldsymbol{\xi} \mid \boldsymbol{\theta}_{g i}\right)=\boldsymbol{\theta}_{g i}{ }^{T} \boldsymbol{\zeta}(\boldsymbol{\xi}),
\end{aligned}
$$


where $\zeta(\xi)=\left(\zeta^{1}(\xi), \ldots, \zeta^{m}(\xi)\right)^{T}$ is supposed to be

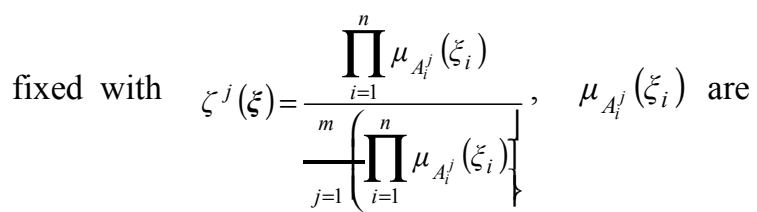
the membership functions of the fuzzy variable $\xi_{i}$, and the vectors $\boldsymbol{\theta}_{f i}{ }^{T}, \boldsymbol{\theta}_{g i}{ }^{T}$ are adaptatively tuned till they reach their optimal value $\boldsymbol{\theta}_{f i}{ }^{*}, \boldsymbol{\theta}_{g i}{ }^{*}$.

\subsubsection{Adaptation Law}

The adaptive functions will be tuned by the next parameter adaptation algorithm (Wang, et al., 2000)

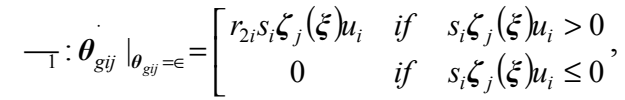

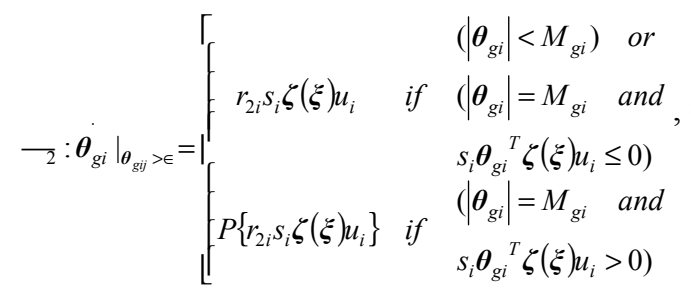

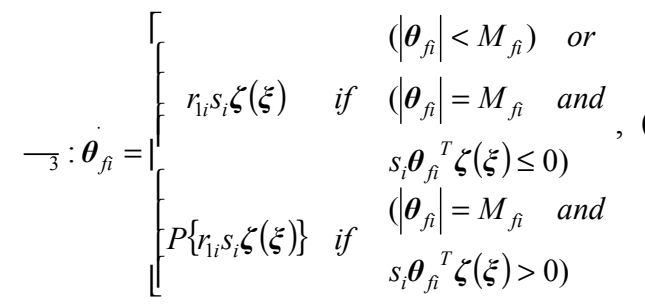

where $r_{1 i}, r_{2 i}, M_{f i}, M_{g i}$ are positive constants specified by the designer, $\zeta_{j}(\xi)$ is the $j^{\text {th }}$ element of $\boldsymbol{\zeta}(\boldsymbol{\xi}), \boldsymbol{\theta}_{g i j}$ is the $j^{\text {th }}$ element of $\boldsymbol{\theta}_{g i}, s_{i}$ and $u_{i}$ are the values of the sliding surface and the control action of the $i^{\text {th }}$ DOF respectively, and the projection operators $P\{*\}$ are defined as $P\left\{r_{1 i} s_{i} \zeta(\xi)\right\}=r_{1 i} s_{i} \zeta(\xi)-r_{1 i} s_{i} \frac{\boldsymbol{\theta}_{f i} \boldsymbol{\theta}_{f i}{ }^{T} \zeta(\xi)}{\left|\boldsymbol{\theta}_{f i}\right|^{2}}$
$P\left\{r_{2 i} s_{i} \zeta(\xi) u_{i}\right\}=r_{2 i} s_{i} \zeta(\xi) u_{i}-r_{2 i} s_{i} \frac{\boldsymbol{\theta}_{g i} \boldsymbol{\theta}_{g i}{ }^{T} \zeta(\xi) u_{i}}{\left|\boldsymbol{\theta}_{g i}\right|^{2}}$. and
Theorem (Wang, et al., 2000) For a nonlinear system (2), consider the controller (4). If the parameter adaptation algorithm (6) is applied, then the system can guarantee that: (a) the parameters are bounded, and (b) closed loop signals are bounded and tracking error converges asymptotically to zero under the assumption of a fuzzy integrable approximation error.

The proof of $\boldsymbol{s}, \boldsymbol{\theta}_{f i}$ and $\boldsymbol{\theta}_{g i}$ boundedness for a SISO system is shown in (Wang, et al., 2000), and similar results could be obtain for the case of MIMO systems, like those presented in (4).

\section{TEST RESULTS}

In this section, the results of a real test are presented in order to show the controller ability to adapt the unknown vehicle dynamic parameters. The test has been carried out in a small tank of $1.8 \mathrm{~m}$ of diameter and $2 \mathrm{~m}$ of depth, by using the Snorkel vehicle with its sensorial an electronic architecture (Sebastian, 2005). Therefore vehicle movements have been limited to yaw rotation, making surge and heave velocities equal to zero. Based on these limits, the test only studies the controller behaviour in yaw movement ( $r$ velocity); however the results can be extended to any other DOF. Finally, it is necessary to point out that the controller equations have been implemented in the vehicle's main CPU, so a digital version of them with an Euler integration algorithm and a sample period of $100 \mathrm{msec}$ has been used.

Prior to developing the test, it is necessary to establish the controller constants based on the theoretical vehicle model (Fossen, 1994) and the boundaries in the parameters uncertainties (Sebastian, 2005). This is the case of the sliding control parameters and the thickness of the boundary layer $b_{r}$ (yaw movement), that have been experimentally fixed.

\begin{tabular}{|c|c|}
\hline PARAMETER & VALUE \\
\hline$\Lambda$ & $\operatorname{diag}\left[\begin{array}{lllll}0.3 & 0.15 & 0.3 & 0.3\end{array}\right]$ \\
\hline$\eta_{4}$ & $\operatorname{diag}\left[\begin{array}{llll}0.38 & 0.13 & 0.19 & 0.55\end{array}\right]$ \\
\hline$b_{r}$ & 1.75 \\
\hline
\end{tabular}

Likewise, the gaussian membership functions associated with each fuzzy estimator have to be defined. Thus, for yaw DOF dependencies have been established.

$$
\hat{f}_{r}=f\left(\mu_{A_{l}}(r), \mu_{A}(u)\right), \quad \hat{g}_{r}=f\left(\mu_{A_{2}}(r)\right)
$$


In table 2, the value of the membership functions parameters can be seen. These functions have to cover all the dynamic range of the kinematic variable, with a certain degree of intersection.

Table 2: Description of membership functions

\begin{tabular}{|c|c|c|}
\hline VARIABLE & $\begin{array}{c}\text { MEMBERSHIP } \\
\text { FUNCTION CENTERS }\end{array}$ & $\begin{array}{l}\text { TYPICAL } \\
\text { DEVIATION }\end{array}$ \\
\hline$\mu_{A_{I}}(r)$ & {$\left[\begin{array}{lllll}-.7 & -.35 & 0 & .35 & .7\end{array}\right] \mathrm{rad} / \mathrm{sec}$} & $0.17 \mathrm{rad} / \mathrm{sec}$ \\
\hline$\mu_{A_{2}}(r)$ & {$\left[\begin{array}{lll}-.7 & 0 & .7\end{array}\right] \mathrm{rad} / \mathrm{sec}$} & $0.35 \mathrm{rad} / \mathrm{sec}$ \\
\hline$\mu_{A}(u)$ & {$\left[\begin{array}{lll}-.5 & 0 & .5\end{array}\right] \mathrm{m} / \mathrm{sec}$} & $0.25 \mathrm{~m} / \mathrm{sec}$ \\
\hline
\end{tabular}

Additionally, the initial values of functions $\hat{f}_{r}$ and $\hat{g}_{r}$ have to be fixed. The initial values of the $\boldsymbol{\theta}_{f i}$ parameters have been initialized to zero, in order to check adaptation capabilities, while $\boldsymbol{\theta}_{g i}$ parameters are set to values that have been obtained based on the vehicle theoretical model (Sebastian, 2005). Finally, some additional constants that determine adaptation behaviour have to be set up, table 3. These constants define functions $\hat{f}_{r}$ and $\hat{g}_{r}$ boundaries, determining the system capability to absorb model perturbations and parameters uncertainties.

Table 3: Adaptive fuzzy control parameters

\begin{tabular}{cccccc}
\hline PARAMETER & $\boldsymbol{M}_{\boldsymbol{f r}}$ & $\boldsymbol{M}_{\boldsymbol{g} r}$ & $\boldsymbol{\varepsilon}_{\boldsymbol{r}}$ & $\boldsymbol{r}_{\boldsymbol{r}}$ & $\boldsymbol{r}_{2 r}$ \\
\hline \multirow{2}{*}{ VALUE } & $2 \cdot \hat{f}_{r_{\max }}$ & $2 \cdot \hat{g}_{r}$ & $\hat{g}_{r} / 2$ & \multirow{2}{*}{0} & \multirow{2}{*}{0.005} \\
& $=1.066$ & $=0.035$ & $=0.0088$ & & \\
\hline
\end{tabular}

To carry out the test a square wave input reference of $10 \% \mathrm{sec}$ of amplitude and $40 \mathrm{sec}$ of period has been used. This input is modified by a first order linear model of reference with a time constant of $1.6 \mathrm{sec}$. The estimation process is kept active thanks to this reference.

After an analysis of the figures, it could be said that the tracking of input reference is almost perfect, in spite of the oscillatory behaviour caused by sensors noise. The estimation of the $\hat{f}_{r}$ function is stable during the test, but some peaks can be observed, whose origin is the oscillatory output around null values of yaw velocity. This effect can be caused by the on line offset adjust algorithm of gyroscopes signals, or by the external system that ensures the correct position of the robot in the middle of the tank. In the same way the estimation of the $\hat{g}_{r}$ function reaches a stable value over the minimum established. The response shows a certain degree of oscillation, which tries to make the value of the sliding surface equal to zero.
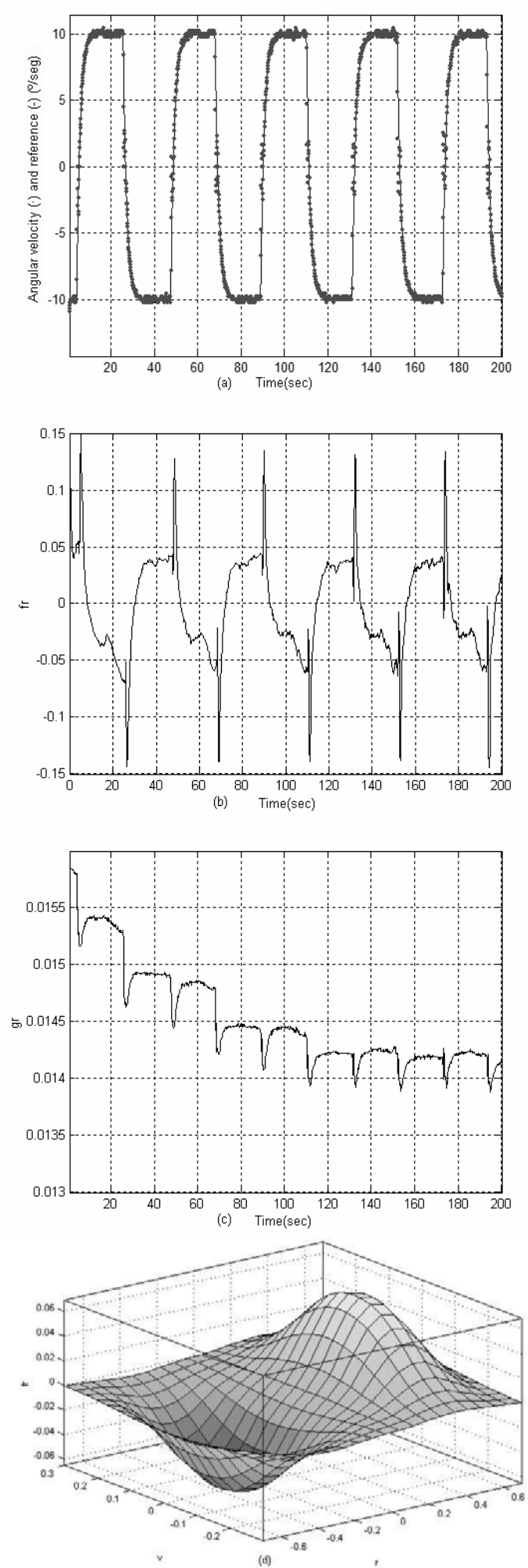

Figure 2: Yaw velocity control test. (a)Yaw velocity $(\cdot)$ and reference (-), (b) $\hat{f}_{r}$ evolution, (c) $\hat{g}_{r}$ evolution and (d)3D representation of $\hat{f}_{r}$. 
Finally, the $3 \mathrm{D}$ representation of $\hat{f}_{r}$ shows the capability of the system to estimate vehicle dynamics, and at the same time, the lack of adaptation for big values of $r$ and non null values of $u$, because the controller has not been tested for them.

\section{CONCLUSIONS}

The adaptive fuzzy sliding mode controller is a valid method to control underwater vehicles, being capable of incorporating the dynamic problems of this type of system, generating designs easily implementable and interpretable, at a reasonable control effort. The theoretical and practical stability of the controller has been demonstrated, ensuring system convergence through reference. Additionally it takes into consideration the nonlinearity of the system and it is capable of adapting to parameters and model uncertainty.

The control proposed can be considered as a combination of an adaptive and robust system. Thus, it has the advantages of both systems. Robust behaviour against fast parameter variation, against perturbations and against noise in the state measurement, are characteristics of the sliding part. On the other hand, no requirement of prior and precise knowledge of uncertainty or its boundaries, and the capability of improving output performance after adaptation, are characteristics of the adaptive part.

The control proposed allows the designer to relax the design conditions of the sliding part, due to the capabilities of the adaptive one to estimate and absorb uncertainties and perturbations. This fact makes possible a reduction in discontinuous control gain, decreasing the chattering the effect.

As a future work, several tests with combined input references and comparisons between the proposed method and existing methods must be carried out. Additionally, tests using the guidance controller have to be developed in order to shown the architecture performance (Sebastian, 2005).

\section{REFERENCES}

Antonelli G., Caccavale F., Chiaverini S. and Fusco G. 2003. A Novel Adaptive Control Law for Underwater Vehicles. IEEE Transactions on Control Systems Technology, 11(2), 109-120.

Choi, S.K. and Yuh, J., 1996. Experimental study on a learning control system with bound estimation for underwater vehicles, International Journal of Autonomous Robots, 3(2/3), 187-194.

DeBitetto, P.A., 1994. Fuzzy logic for depth control for unmanned undersea vehicles, Symposium of Autonomous Underwater Vehicle Technology. Cambridge, MA, 233-241.

Espinosa F., López E., Mateos R., Mazo M. and García R. 1999. Application of advanced digital control techniques to the drive and trajectory tracking systems of a wheelchair for the disabled. Emerging Technologies and Factory Automation, Barcelona, 521-528.

Fossen, T. I., 1994 Underwater vehicle dynamics. Baffins Lane, Chichester, John Wiley \& Sons Ltd.

Gee S.S., Hang C.C. and Zhang T. 1999. A direct method for robust adaptive nonlinear with guaranteed transient performance. Systems and Control Letters, 37, 275284.

Sebastián, E. 2005. Control y navegación semi-autónoma de un robot subacuático para la inspección de entornos desconocidos. Doctoral diss., Universidad de Alcalá, Madrid.

Slotine, J.J. and Li W., 1991. Applied nonlinear control. Englewood Cliffs. Prentice Hall.

Wang, J., Get S.S. and Lee T. H., 2000. Adaptive Fuzzy Sliding Mode Control of a Class of Nonlinear Systems. $3^{\text {rd }}$ Asian Control Conference, Shanghai. 599-604.

Yoerger, D.R. and Slotine J.E., 1991. Adaptive sliding control of an experimental underwater vehicle. IEEE International conference on Robotics and Automation, Sacramento. CA. 2746-2751.

Yuh, J., 1994. Learning control for Underwater Robotics Vehicles. IEEE Control System Magazine. 14(2) 39-46.

Yuh J. 2000. Design and Control of Autonomous Underwater Robots: A Survey. Autonomous Robots, 8 , 7-24. 